\title{
The Water Cherenkov Detector Array of the LAGO project in Huancayo - Peru
}

\author{
L. Otiniano*, Ch. Alvarado, W. Guevara, J. Truyenque, F. Quispe \\ Dirección de Astrofísica Comisión Nacional De Investigación y Desarrollo Aeroespacial \\ Lima, Perú \\ E-mail: lotinianodconida.gob.pe

\section{for The LAGO Colllaboration} \\ lagoproject.org/collab.html
}

\begin{abstract}
One importan goal of the High Energy program of the LAGO project is to detect the high energy component of cosmic rays due to Gama Ray Bursts. Therefore, high altitude sites (over 4500 m.a.s.l) are chosen for installation of some detectors. The altitude allows the improvement of the sensitivity of the detectors otherwise reduced due to the strong absorption of extensive air showers initiated by gamma rays in the atmosphere.

In Peru, near the city of Huancayo $\left(12^{\circ} 2,7^{\prime} S 75^{\circ} 20.4^{\prime} \mathrm{W}\right)$, an array of three water Cherenkov detectors for the LAGO project is under construction. Here we describe the chosen location for this purpose and the detector development. Then, we show the development of a semi-analytical method for fast calibration and its comparison to CORSIKA/Geant4 simulations.
\end{abstract}

35th International Cosmic Ray Conference - ICRC2017

10-20 July, 2017

Bexco, Busan, Korea

${ }^{*}$ Speaker. 


\section{Introduction}

The LAtinoamerican Giant Observatory (LAGO) international collaboration High Energy program goal is to observe high energy component (over $10 \mathrm{GeV}$ ) of Gamma Ray Bursts (GRBs) using individual or small arrays of Water Cherenkov Detectors (WCD) at high altitude sites [1]. When a GRB reach the top of the atmosphere a multitude of high energy gamma rays produces decay showers. Together those showers could be detectable at ground level as an instant excess in the flux measured in a detector or array of detectors. This showers contend 99\% of electromagnetic particles WCDs are sensitive to this particles. As these showers are strongly absorbed by the atmosphere, the WCDs must be placed at high altitude in order to detect the greatest possible number of particles.

In Peru a new array of the WCDs for the LAGO project is under development[5]. In this article we describe the site chosen for this purpose and the detector prototype, then we describe a GEANT4[2] simulation of the detector and his validation using data of the prototype.

\section{Huancayo Detector}

The Detector is located at Huayao Magnetic Observatory (OMH, $\left.12{ }^{\prime \prime} 02.7^{\prime} \mathrm{N} 75^{\prime \prime} 20.4^{\prime} \mathrm{W}\right)$ in the Central Sierra of Peru on a vast plain at 3300 m.a.s.l. For monitoring detectors and storage of materials a mobile station has been built using steel containers.

The LAGO WCD is commonly made of a water commercial tank, filled with filtered and chemically purified water, a external coberture for light isolation and an internal liner of Tyvek, to reflect and diffuse Cherenkov photons produced in water by high energy secondary cosmic rays. The diffusion of Cherenkov photons reduces the signal dependence with the secondary particle trajectory. A large area photomultiplier PMT complete the detector[1]. As our ultimate goal is the installation of detectors at high altitude we developed a prototype made of a cylindrical surface ( $2.5 \mathrm{~m}$ diameter $1 \mathrm{~m}$ height) assembled using four stainless steel plates that support an internal cylindrical pool filled with treated water, inside the pool a PVC pipe structure supports a cylindrical bag of Tyvek an upper 9354KB Electron Tube (PMT), see Figure 3. For light isolation we use six layers of low density black polyethylene $(.1 \mathrm{~mm})$ and a layer of $0.25 \mathrm{~cm}$ of high density black polyethylene (which also protects the environment detector).

The typical signal produced by our detector by crossing particles is a negative pulse with a fast decay and an exponential recovery, the pulse amplitude is $100 \mathrm{mV}$ and width of $100 \mathrm{~ns}$. The LAGO project has development its own DAQ system with three channels at $40 \mathrm{MHz}$ sampling rate 10 bits digitalization (ADC unit $\approx 1 \mathrm{mV}$ ), time stamp via GPS signal, sampling of pressure and temperature each second and control for high voltage powers supply needed to operate and record the signal of PMTs [9].

\section{Detector Response Simulation}

Using the LAGO collaboration tools, CORSIKA[3] based, we simulate radiation environment of secondary cosmic rays [1]. Corrected for geomagnetic effects by cutting the spectrum of primaries reaching the atmosphere [4]. Figure 1 shows the result of simulation spectrum of secondary 
cosmic rays for the $\mathrm{OMH}$ at 3300 m.a.s.l. Then we use GEANT4 simulation of the dedector to validate and estimate the response of detector.

\section{Calibration Method}

For calibration of our detector we need to estimate the value of the charge left by VEM (Veritcal Equivalente Muon) traversing the detector. In [11] we elaborate a process to infer directly from the charge spectrum of secondary particles that cross the detector (figure 1) the value of the VEM, this is done first making a muon selection in the pulses measured. Following [10] we compare two consecutive pulses and discard the one with a smaller peak, after which the spectrum of shit $F(q)$ is transformed by:

$$
F(q) \longrightarrow F(q)+\frac{q}{n} F^{\prime}(q) \propto V^{V E M}(q)
$$

being $V^{V E M}(q)$ the charge distribution of the VEM, Figure 2.

We used this VEM charge distribution to adjust the parameters of our GEANT4 simulation and way to validate the method we calculated, using as inputs the results of CORSIKA background simulation (figure 1), the charge histogram that would leave the total flow of muons of 1 hour when traversing the detector, and compare it with our muon selection, Figure 2.

\section{Discussion and Conclusions}

Our previous prototype detector [6] had light leakage which was noticeable in appreciable variations (day/night) of the charge spectrum, in Figure 1 we show these histograms at noon and midnight which do not show appreciable differences, this gives us confidence in our design (Figure $3)$.

The calibration process is under development, it is necessary to refine the simulation, we have not been able to reproduce the spread in the VEM or muon histogram, we believe that is caused by the water contamination. We manage to adjust the simulation to reproduce the VEM peak and the muon peak on charge histograms (Figure 2)

\section{Acknowledgments}

The autors gratefully acknowledges the financial support from Fondo para la Innovación, Ciencia y Tecnología (FINCyT) trough the project PIBA-2-P-020-14. The LAGO Collaboration is very thankful to the Pierre Auger Collaboration for its continuous support.

\section{References}

[1] H. Asorey for the LAGO Collaboration. LAGO: the latin american giant observatory. Nuclear Instruments and Methods in Physics Research Section A: Accelerators, Spectrometers, Detectors and Associated Equipment, 2017.

[2] S. Agostinelli, J. Allison, K. Amako, J Apostolakis, H. Araujo, P. Arce, M. Asai, D Axen, S. Banerjee, G. Barrand, et al. Geant4: a simulation toolkit. Nuclear instruments and methods in physics research section A: Accelerators, Spectrometers, Detectors and Associated Equipment, 506(3):250-303, 2003. 
[3] D. Heck et al. CORSIKA : A Monte Carlo Code to Simulate Extensive Air Showers. FZKA,6019:1-98, 1998.

[4] M.S. Durán, H. Asorey, S. Dasso, L. Nuñez, Y. Peréz, C. Sarmiento for the LAGO Collaboration. The LAGO space weather program: Directional geomagnetic effects, background fluence calculations and multi-spectral data analysis, The 34th International Cosmic Ray Conference Vol. PoS (ICRC2015) (p. 142).

[5] Otiniano, L., et al. Development of a high altitude lago site in Peru. The 34th International Cosmic Ray Conference, Vol. PoS (ICRC2015). (p. 688).

[6] Tueros-Cuadros, et al. The LAGO (Large Aperture GRB Observatory) in Peru. Proceedings of the International Astronomical Union volume 7(S286), 445-447,2011

[7] Etchegoyen, A., et al. Muon-track studies in a water Cherenkov detector. Nuclear Instruments and Methods in Physics Research Section A: Accelerators, Spectrometers, Detectors and Associated Equipment 2005, vol. 545, no 3, p. 602-612.

[8] L. Otinino, A., et al. VEM Semianalitycal Correction in LAGO water Cherenkov detector. In preparation

[9] Haro, M. Sofo, L. H. Arnaldi, et al. The data acquisition system of the Latin American Giant Observatory (LAGO). Nuclear Instruments and Methods in Physics Research Section A: Accelerators, Spectrometers, Detectors and Associated Equipment 820 (2016): 34-39.

[10] Salazar, H., and L. Villasenor. Separation of cosmic-ray components in a single water Cherenkov detector. Nuclear Instruments and Methods in Physics Research Section A: Accelerators, Spectrometers, Detectors and Associated Equipment 553.1 (2005): 295-298.

[11] L. Otiniano, et al. The cosmic rays web monitor of the LAGO project. This Procedings, volume in press, 2017.
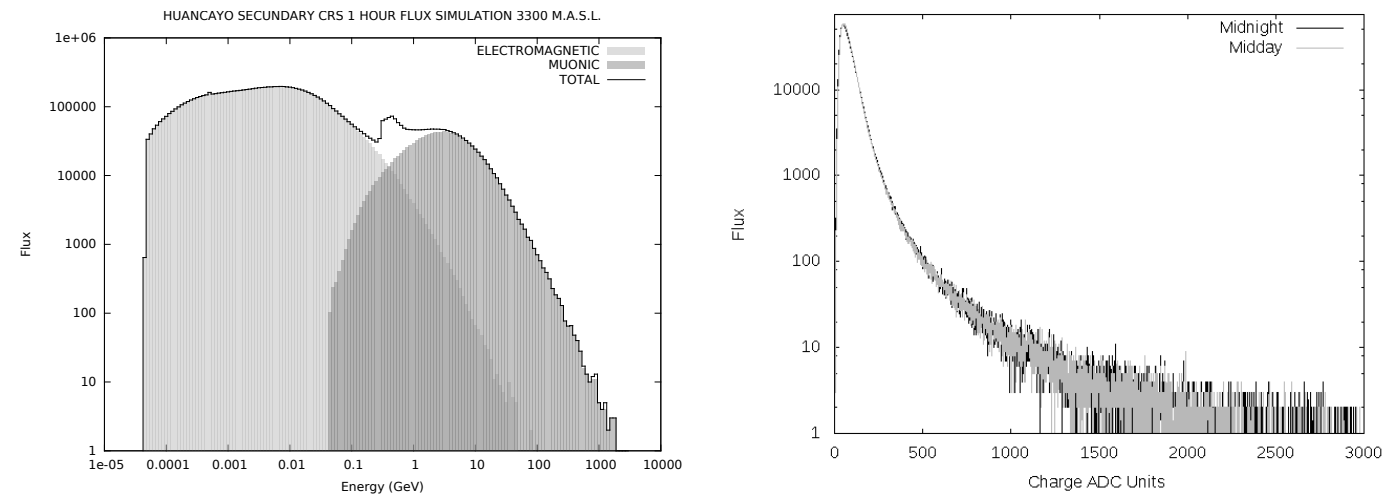

Figure 1: Simulated secondary cosmic ray spectrum in the location of the $\mathrm{OMH}$ at 3300 m.a.s.l. we show the main components of the flux (electromagnetic and muon) (left). One Hour charge histogram measured on the detector at middy and midnight. No appreciable differences are notice (right) 

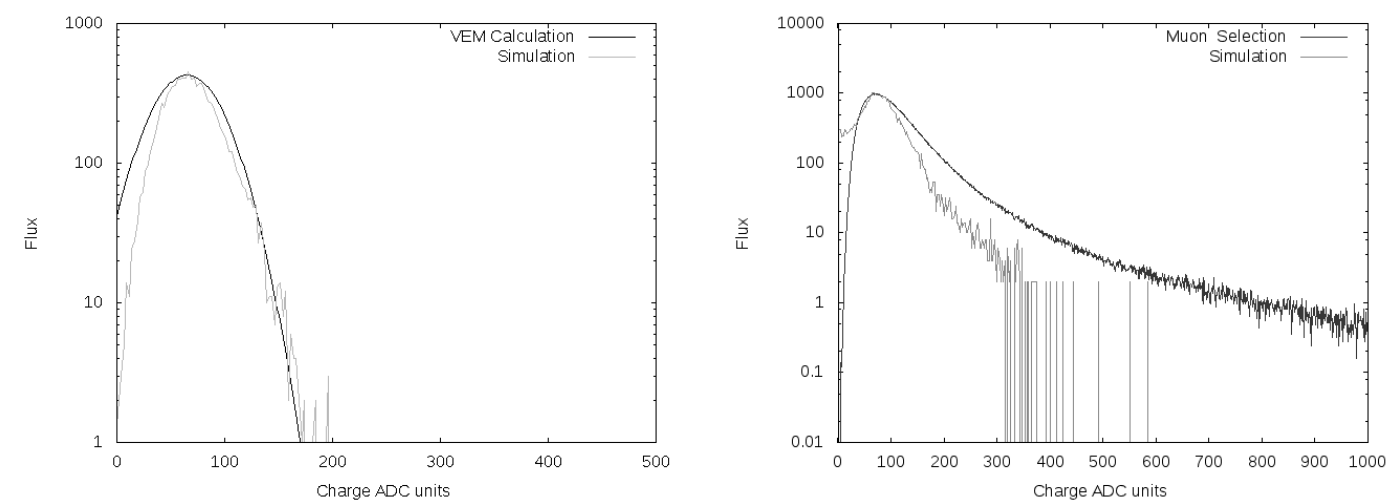

Figure 2: VEM charge histogram calculated from total charge and simulated (20000 particles) (left). Muon selection charge histogram calculated from total charge and simulated (40000 particles) (right). To be able to compare the calculated spectra were normalized

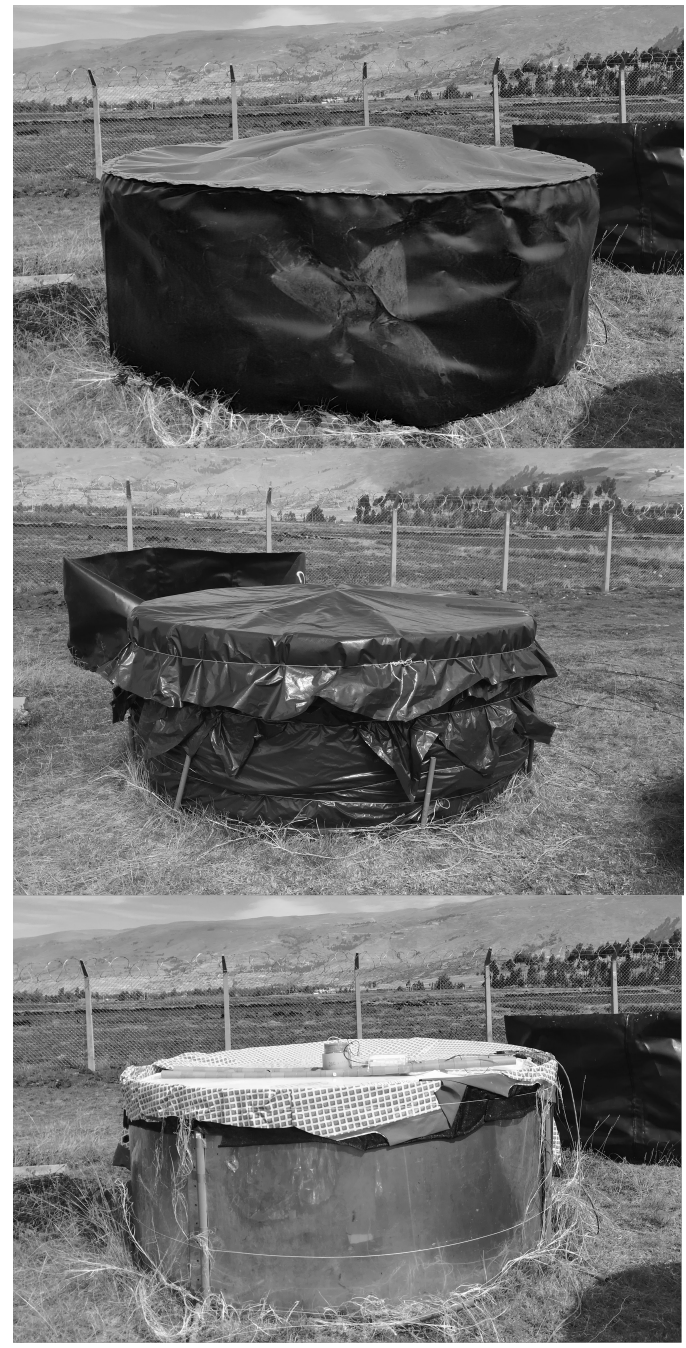

Figure 3: From botton to top: 1) Cylindrical metal pool, Tyvek bag and sensor. 2) Low density black polyethylene coating. 3) high density black polyethylene 